\title{
亚胺引发四肽环化反应机理的理论研究
}

\author{
田燕 ${ }^{a, b} \quad$ 傅尧 ${ }^{a} \quad$ 张琪 $^{a}$ 于海珠*, $a$ 石景*, \\ ( ${ }^{a}$ 中国科学技术大学化学系 合肥 230026) \\ ( ${ }^{b}$ 安徽农业大学应用化学系 合肥 230036)
}

\begin{abstract}
摘要 运用密度泛函的理论方法(M06//B3LYP)对亚胺引发的关环肽环化的反应机理进行了研究. 计算结果表明, 由亚 胺到环肽的转化过程存在两种不同的反应机理. 一种是羰基转移一合环机理: 先经历羰基转移, 然后 H转移与 C-O 合环 同时进行. 另一种是 $\mathrm{H}$ 转移-合环-羰基转移- $\mathrm{H}$ 转移机理: $\mathrm{H}$ 原子先从 $\mathrm{O}$ 原子转移到 $\mathrm{N}$ 原子然后再进行 $\mathrm{C}-\mathrm{O}$ 合环, 随后 再进行羰基转移与 $\mathrm{H}$ 原子转移. 计算结果显示, 羰基转移-合环反应历程为有利路径, 该反应路径中的 $\mathrm{H}$ 原子转移及 $\mathrm{C}-\mathrm{O}$ 成环过程为整个反应的决速步骤.
\end{abstract}

关键词 环肽; 协同反应; 分步反应; 反应机理; 密度泛函理论

\section{Theoretical Study on the Mechanism of the Imine-induced Tetrapeptides Cyclization}

\author{
Tian, Yan ${ }^{a, b} \quad \mathrm{Fu}, \mathrm{Yao}^{a} \quad$ Zhang, $^{\mathrm{Qi}^{a}} \quad \mathrm{Yu}, \mathrm{Haizhu}^{*, a} \quad$ Shi, Jing*,a \\ $\left({ }^{a}\right.$ Department of Chemistry, University of Science and Technology of China, Hefei 230026) \\ ( ${ }^{b}$ Department of Applied Chemistry, Anhui Agricultural University, Hefei 230036)
}

\begin{abstract}
The small-sized cyclic peptides have attracted much research interest in the organic and bioorganic chemistry. In the present study, the mechanism of cyclization of tetrapeptides by an imine-induced ring-closing strategy has been studied in detail using the density functional theory method. The geometries of all the species were fully optimized at the B3LYP/6-31G(d) level. The M06/6-311++G(2df,2p) method is employed to calculate the single point energies. The solvent effect [Trifluoroacetic Acid (TFA) was used as a solvent] was calculated by using SMD (solvation model based on the quantum mechanical charge density of a solute molecule interacting with a continuum description of the solvent) model. Both the carbonyl transfer-cyclization and the $\mathrm{H}$ transfer-cyclization-carbonyl transfer-H transfer mechanism might be responsible for the transformation of the imine reagent to the cyclic peptide product. According to the calculation results, the carbonyl transfer-cyclization mechanism occurs via the carbonyl transfer from $\mathrm{O}(\mathrm{Ph}-)$ atom to $\mathrm{N}$ atom, corresponding to three possible transition states with the barrier height of $20.2,16.4$ and $62.7 \mathrm{kcal} / \mathrm{mol}$, respectively (the second one is the most feasible among different ones). Then the concerted $\mathrm{H}$ transfer \& $\mathrm{C}-\mathrm{O}$ cyclization step occurs via a six-membered ring transition state. The second step (the concerted $\mathrm{H}$ transfer \& $\mathrm{C}-\mathrm{O}$ cyclization step) is the rate-determining step, and the overall activation barrier is $28.3 \mathrm{kcal} / \mathrm{mol}$. In contrast, the $\mathrm{H}$ transfer-cyclization-carbonyl transfer-H transfer mechanism includes four steps: the hydrogen atom first transferred from oxygen atom to $\mathrm{N}$ atom with the breaking of $\mathrm{C}-\mathrm{C}$ to form the intermediate $\mathbf{M} 3$ and a $\mathrm{CH}_{3} \mathrm{COH}$ molecule, cyclization via the $\mathrm{C}-\mathrm{O}$ and $\mathrm{C}-\mathrm{C}$ bond formation, the carbonyl transfer from $\mathrm{O}(\mathrm{Ph}-)$ atom to $\mathrm{N}$ atom accompanied with the breaking of $\mathrm{C}-\mathrm{N}$, and the transfer of hydrogen atom with the cyclization of $\mathrm{C}-\mathrm{N}$ then occurred subsequently. In this context, the second step (i.e. cyclization via the $\mathrm{C}-\mathrm{O}$ and $\mathrm{C}-\mathrm{C}$ bond formation) is the rate-determining step, and the overall activation barrier is $40.1 \mathrm{kcal} / \mathrm{mol}$. Accordingly, the carbonyl transfer-cyclization mechanism is relatively more feasible than the $\mathrm{H}$ transfer-cyclization-carbonyl transfer- $\mathrm{H}$ transfer mechanism, and the rate-limiting step is the concerted $\mathrm{H}$ transfer \& C-O cyclization step with a barrier of $28.3 \mathrm{kcal} / \mathrm{mol}$.

Keywords cyclic peptide; concerted reaction; stepwise reaction; reaction mechanism; density functional theory
\end{abstract}

\section{1 引言}

环肽是一类重要的肽类化合物, 其由于存在特殊的
环状结构而具有较高的稳定性. 环肽一般具有广泛的生 理活性，因此这类物质在生物医学、药学等领域都具有 巨大的发展前景 ${ }^{[1]}$. 环肽源于人们对天然产物组分的研

*E-mail: shijing@ustc.edu.cn; yuhaizhu@gmail.com; Tel.: 0551-63607476; Fax: 0551-63606689

Received May 21, 2014; published June 24, 2014.

Supporting information for this article is available free of charge via the Internet at http://sioc-journal.cn.

Project supported by the National Natural Science Foundation of China (Nos. 21325208, 21172209, 21361140372, 21202006), the Specialized Research Fund for the Doctoral Program of Higher Education (No. 20123402110051), the Fundamental Research Funds for the Central Universities (No. WK2060190008), Chinese Academy of Sciences (No. KJCX2-EW-J02) and the ChinaGrid project funded by MOE of China, and the super-computer center of Shanghai. 项目受国家自然科学基金(Nos. 21325208, 21172209, 21361140372, 21202006)、高等学校博士学科点专项科研基金资助课题(No. 20123402110051)、中 国科学院基金(No. KJCX2-EW-J02)、中央高校基本科研业务费专项基金(No. WK2060190008)及教育部中国教育科研网格 ChinaGrid 及上海超级计算 中心资助. 
究, 20 世纪 40 年代第一个抗菌类环肽 gramicidin $\mathrm{S}$ 被发

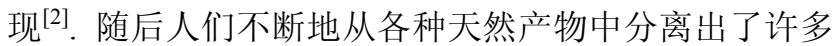
不同生理活性的环肽. 首尾相连的环肽, 其环状结构具 有一定的构象约束作用, 其抗酶解能力比线性肽强 ${ }^{[3]}$. 这一特点使环肽具有许多重要的性质, 其结构特点及在 药学上的优势也引起了化学家的广泛兴趣 ${ }^{[4]}$. 化学合成 对研究多肽/蛋白质分子的构效关系有着巨大的作用. 与线性肽相比, 运用化学合成的手段来实现环肽的合成 要困难的多. 环化反应属于分子内反应, 需要在高度稀 释的溶液中进行, 但仍然会存在二聚体及多聚体的生

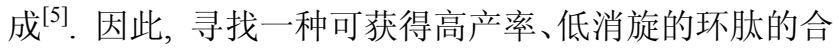
成方法已经成为当今化学家的研究热点之一 ${ }^{[6]}$.

最近, Wong 等 ${ }^{[7]}$ 报道了一个由亚胺引发关环来合 成首尾相连的四肽的实验方法, 这一路线应用了 $\mathrm{Li}$ 等 ${ }^{[8]}$ 发展的新型丝氨酸介导的多肽连接反应. 该方法拓展了 传统的半胱氨酸介导的自然连接反应 ${ }^{[9]}$. 在环肽合成这 一应用中, 他们指出由亚胺到四肽的环化过程可能存在 两种不同的反应历程. 如图式 1 所示, 他们认为在整个 合成反应中由水杨酸脂肽 $\mathbf{A}$ 出发会优先生成一个首尾 相连的 16 元环的亚胺物质 $\mathbf{B}$, 随后再经过一个羰基转移 的中间体 $\mathbf{C 1}$ 或者羟基-烯烃合环的中间体 $\mathbf{C 2}$, 再生成 一个 12 元环的 $N, O$-苯亚甲基的缩醛中间物质 $\mathbf{D}$, 最后 物质 $\mathbf{D}$ 经过酸解生成最终的 12 元环的环肽 $\mathbf{E}$. 同时, 他 们也指出由 $\mathbf{B}$ 到 $\mathbf{D}$ 的过程中会有四个价键的生成, 由于 物质 B 自身的结构决定了似乎并不能有效地降低环收 缩过程的应变能. Wong 等运用理论计算对底物中胺基 进攻醛基及酯基的能垒进行估算, 并由此推断 $\mathrm{B} \rightarrow \mathrm{C} 2$ 过程会优先发生.

应该注意到, Wong 小组仅对这两个反应路径中的 胺基进攻步骤进行研究, 因此目前仍缺少对整个肽环化 反应机理细节的认识. 此外, Wong 等的计算中运用 $\mathrm{NH}_{2}$ 基团替代图式 1 所示反应体系内的亚胺基团, 其可行性 仍有待商榷. 因此, 由亚胺 $\mathbf{B}$ 到环化中间体 $\mathbf{D}$ 的转化过 程还需要进一步的研究, 进而明确其内在的反应机理, 并为实验提供更好的参考和辅助设计作用. 为此, 我们 在本文中运用理论计算方法对四肽合成中的关键部分 的反应历程进行详细研究, 揭示其内在的反应机理. 基

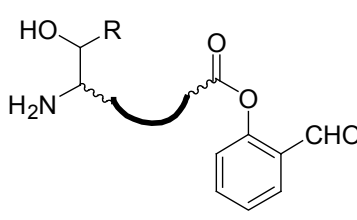

A r.t., $4 \mathrm{~h}$

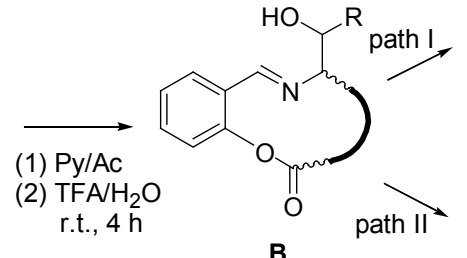

B
于对模型体系的简化, 我们选取 Eq. 1 为模型化反应, 运用密度泛函理论(DFT)方法对由反应物 $\mathbf{R}$ (对应图式 1 中的物质 B) 出发生成产物 $\mathbf{P}$ (对应图式 1 中的物质 $\mathbf{D}$ )的 两个不同的机理进行详细的研究.<smiles>[R]C(=O)Oc1ccccc1/C=N/C(C)C(C)O</smiles>

\section{2 计算方法}

密度泛函的 B3LYP 理论方法 ${ }^{[10]}$ 能够给出比较准确 的分子结构信息 ${ }^{[11]}$, 已经被成功的用于描述生物分子 的相互作用 ${ }^{[12]}$. 本文中所有物质在气相中的几何结构 优化均是在 B3LYP 理论水平下以 6-31G(d)基组进行的 (采用 M06泛函进行构型优化得到的结果与 B3LYP 一致, 计算结果见 Supporting information). 在同等理论水平上 对所有优化构型进行频率分析, 以确定反应物、中间体 和产物均不存在任何虚频为势能面上的极小点, 而过渡 态仅存在一个虚频，为势能面上的鞍点. 同时，每一个 鞍点还进行了內禀反应坐标 $(\mathrm{IRC})^{[13]}$ 分析, 来确认其在 势能面上连接着正确的反应物和产物. 最后运用密度泛 函理论中的 M06 ${ }^{[14]}$ 方法以 6-311++G(2df,2p)为基组对 各稳定物质的单点能进行计算. 为了验证 M06 能量的 可靠性, 除此之外, 我们还分别运用 $\mathrm{TPSS}^{[15]}$ 及 MPWB1 $\mathrm{K}^{[16]}$ 的方法对反应体系中各物质的单点能进行 计算. 在计算单点的同时用建立在溶质与溶剂相互作 用的电荷密度基础上的溶剂化模型 $(\mathrm{SMD})^{[17]}$ 对溶剂 化效应(以 $\mathrm{CF}_{3} \mathrm{COOH}$ 为溶剂)进行计算. 本文中给出的 所有物质的能量均是用气相优化得到的吉布斯自由能 的矫正值与考虑了溶剂效应后的单点能相加得到的结 果. 所有的计算均是用高斯 09 程序包 ${ }^{[18]}$ 完成的.

\section{3 结果与讨论}

首先对初始反应物模型 $\mathbf{R}$ 进行构型优化, 在优化

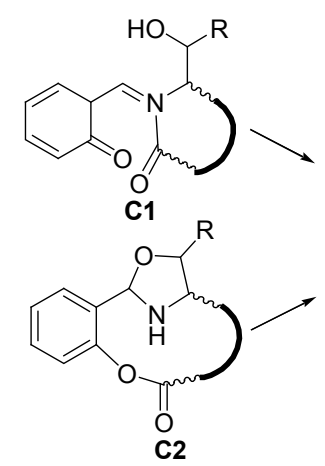

图式 1 四肽环化过程中两种可能的反应历程

Scheme 1 Two possible pathways of the proposed tetrapeptide cyclization 
得到的几个空间异构体(详见 Supporting information)中 选取其中能量相对较低的一个 (记为 $\mathbf{R}$ ) 作为本体系的零 参考点. 本文中其余物质的相对能量均是以 $\mathbf{R}$ 为参考点 得到的相对能量.

\section{1 羰基转移一合环反应机理}

首先研究了由反应物 $\mathbf{R}$ 出发先进行酚酯中的羰基 基团转移到 $\mathrm{N}$ 原子的过程, 也就是图式 1 中的由 $\mathbf{B}$ 到 C1 的过程(path I). 我们设想, 在反应路径上可能会存 在一个分子内酰基转移过程 ${ }^{[19]}$ 的 6 元环的中间体 M1, 如 Eq. 2 所示. 可是始终没有优化得到 M1 的稳定构型, 每次都是以得到与初始反应物 $\mathbf{R}$ 相似的构型而失败. 计 算结果显示, 由 $\mathbf{R}$ 出发会直接经历一个酰基转移的过渡 态 TS1 而生成中间体 M2-1(其优化构型如图 1 所示). M2-1 即为图式 1 所示 path I 中的中间体 C1. 如图 1 所 示, 在 TS1 中要断裂的 $\mathrm{C}-\mathrm{O}$ 键被拉伸到了 $1.791 \AA$, 而 即将成键的 $\mathrm{C}-\mathrm{N}$ 之间的距离缩短至 $1.610 \AA$. 以上结构 数据表明, 在酰基转移过程中, 羰基 $\mathrm{C}-\mathrm{O}(\mathrm{Ph}-\mathrm{O})$ 键的 断裂和羰基 $\mathrm{C}-\mathrm{N}$ 键的生成协同进行. 在图 2 中给出了 该反应过程的相对自由能势能曲线. 相关数据表明此过 程的自由能垒为 $20.2 \mathrm{kcal} / \mathrm{mol}$.<smiles>[R]C(=O)Oc1ccccc1/C=N/C(C)C(C)O</smiles>

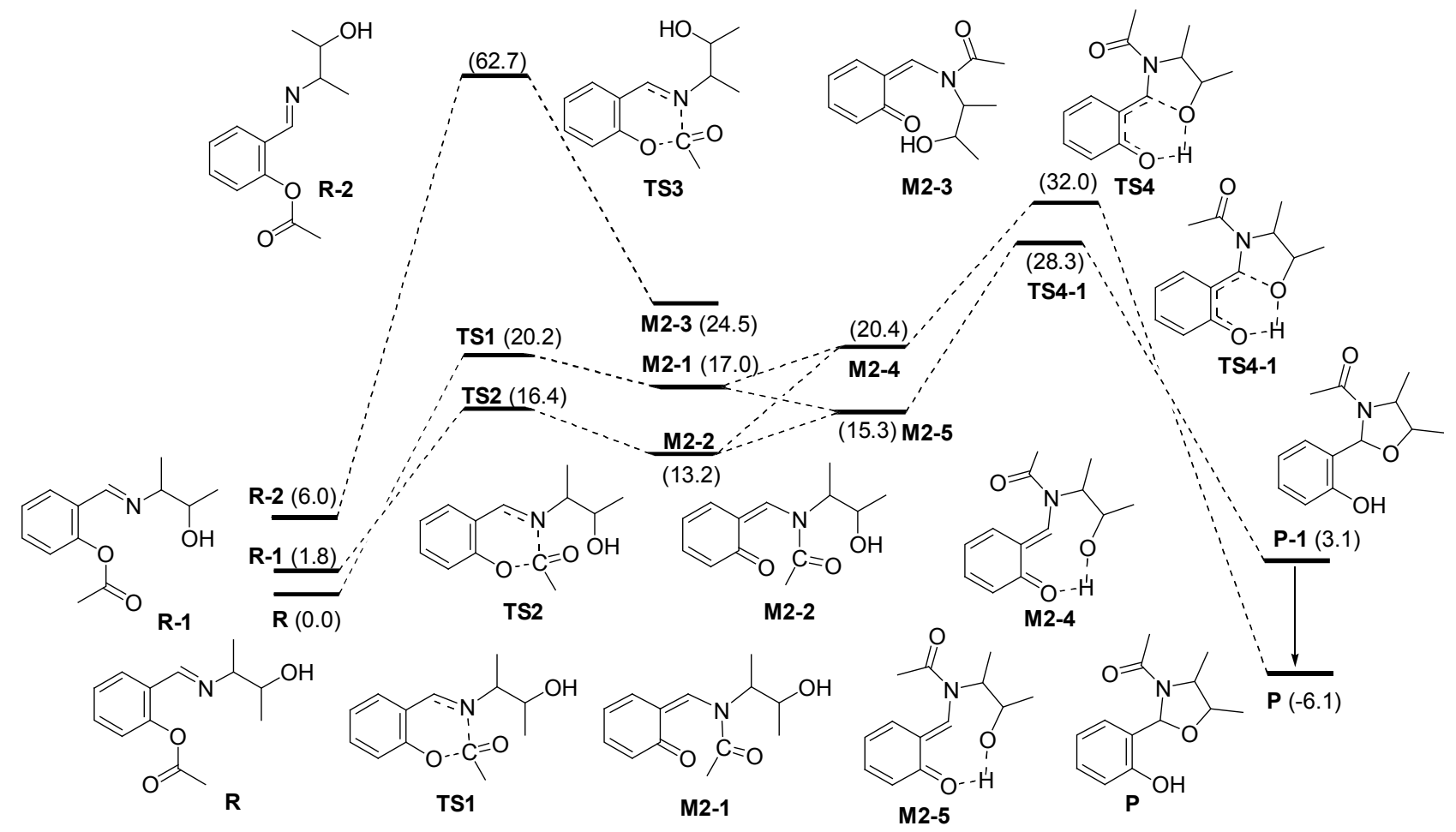

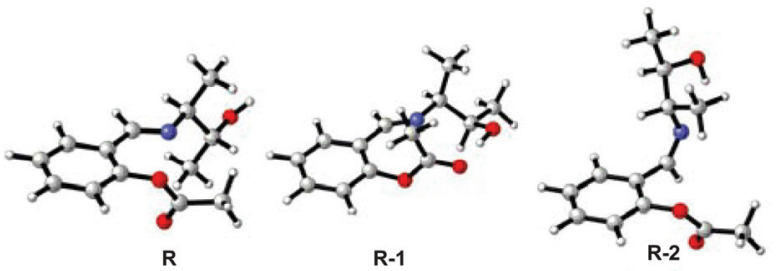
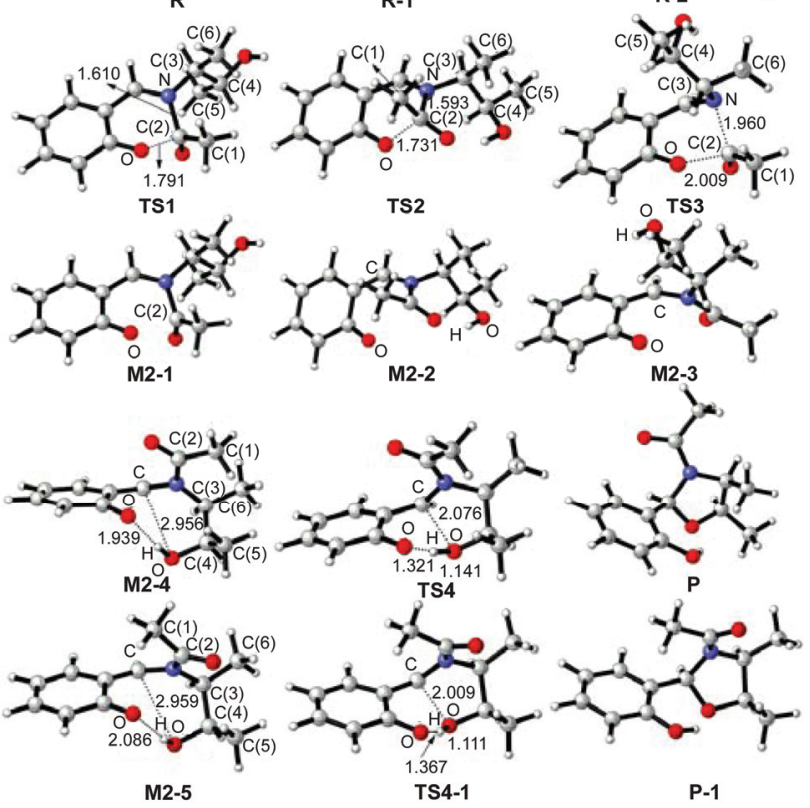

图 1 羰基转移-合环反应中各物质的优化构型(键长单位为 $\AA$ )

Figure 1 Optimized structures of species in the carbonyl transfer- cyclization reaction mechanism (the bond lengths are given in $\AA$ )

图 2 羰基转移一合环反应中的势能面图(图中的数据为吉布斯自由能, 单位 $\mathrm{kcal} / \mathrm{mol}$ )

Figure 2 Energy profile of carbonyl transfer-cyclization reaction (the values in profile are Gibbs free energies, in kcal/mol) 
随后，在 TS1 构型的基础上对末端的甲基基才绕着 $\mathrm{C}(3)-\mathrm{C}(4)$ 键旋转及调整 $\mathrm{C}(5)-\mathrm{C}(4)-\mathrm{C}(3)-\mathrm{C}(6)$ 二面角又 优化得到了另外两个不同的羰基转移过程的过渡态 TS2 和 TS3, 它们的相对自由能值分别为 16.4 和 62.7 $\mathrm{kcal} / \mathrm{mol}$. 如图 1 所示, 二面角 $\mathrm{C}(1)-\mathrm{C}(2)-\mathrm{N}-\mathrm{C}(3)$ 和 $\mathrm{C}(5)-\mathrm{C}(4)-\mathrm{C}(3)-\mathrm{C}(6)$ 分别由 TS1 中的 $61.2^{\circ},-170.2^{\circ}$ 变 化到 TS2 中的 $109.2^{\circ}$ 和 $-78.2^{\circ}$. 同时, TS2 中要断裂的 $\mathrm{C}$ - $\mathrm{O}$ 之间的距离被拉伸到 $1.731 \AA$, 而将要成键的 $\mathrm{C}-$ $\mathrm{N}$ 的长度已被缩短至 $1.593 \AA$. 除此之外, 二者在空间结 构上并没有太大的变化, 因此, 二者在自由能方面也仅 相差了 $3.8 \mathrm{kcal} / \mathrm{mol}$. 与 TS1, TS2 相比, TS3 在空间结构 上有了较大的变化. 如图 1 所示, TS3 是在 TS1 的基础 上通过连在 $C(3)$ 原子上的 $C(6)$ 与 $C(4)$ 基团绕着 $C(3)-N$ 轴旋转得到的, 其结果会增加羰基转移的空间位阻. 因 此, 在 TS3 中要断裂的 C-O 键被拉伸到了 $2.009 \AA$, 而 即将成键的 $\mathrm{C}-\mathrm{N}$ 之间的距离仅缩短至 $1.960 \AA$. 相应的, 通过 TS3 生成中间体 M2 的自由能垒高达 $62.7 \mathrm{kcal} / \mathrm{mol}$. 由于 TS2, TS3 与 TS1 三者在空间结构上存在差异, 在 接下来的 IRC 分析发现 TS2 和 TS3 所连接的初始反应 物与产物并不是 $\mathbf{R}$ 和 $\mathbf{M} 2-1$, 而是二者的异构体, 分别 记为 R-1 与 M2-2 和 R-2 与 M2-3. 由图 2 可以得出, 羰 基转移过程最有利的路径所对应的自由能垒为 16.4 $\mathrm{kcal} / \mathrm{mol}$. 此结果稍高于 Wong 等 ${ }^{[7]}$ 的计算结果 14.8 $\mathrm{kcal} / \mathrm{mol}$. 其原因是由于他们在计算中运用 $\mathrm{NH}_{2}$ 基团替 代图式 1 所示反应体系内的亚胺基团, 这在一定程度上 降低了羰基转移的空间位阻作用. 图 2 中的数据表明, 由反应物 $\mathbf{R}$ 经历三个不同的过渡态生成中间体 $\mathbf{M} 2$ 均会 引起体系的吉布斯自由能升高, 此结果与 Wong 小组 ${ }^{[7]}$ 的计算结果相一致.

如图 1 所示，中间体 M2-1 及 M2-2 中羟基与烯酮的 酮键距离较远难以直接发生 $\mathrm{H}$ 原子转移及 $\mathrm{C}-\mathrm{O}$ 合环而 生成产物. 因此, 由中间体 M2-1 及 M2-2 出发, 先经历 几个单键旋转的异构化过程生成中间体 M2-4 和 M2-5. 其中羟基基团的空间取向都有利于接下来进行的 $\mathrm{H}$ 原 子的转移及 C-O 合环过程的发生. 随后 M2-4 与 M2-5 均经历一个羟基的 $\mathrm{H}$ 原子转移到酚羟基 $\mathrm{O}$ 原子及 $\mathrm{C}-\mathrm{O}$ 成环协同进行的过渡态而生成产物 $\mathbf{P}$. 二者结构上的主 要区别在于 $\mathrm{N}$ 原子上连接的羰基基团在空间伸展方向 的不同. 因此, 在接下来的反应路径中 M2-4 直接经历 过渡态 TS4 而生成终产物 P. 而 M2-5 先经历过渡态 TS4-1 生成产物 P-1, 然后再经过单键旋转的方式异构 化为稳定的终产物 P. 如图 1 所示, 在 M2-4 中 C(1)的甲 基基团距离 $\mathrm{C}(6)$ 的甲基基团较近，由于空间位阻的排斥 作用使得 M2-4 的自由能比 M2-5 的高出 $5.1 \mathrm{kcal} / \mathrm{mol}$. 从而也导致过渡态 TS4 的自由能比 TS4-1 的高 3.7 $\mathrm{kcal} / \mathrm{mol}$. 综上所述, 在整个羰基转移一合环反应过程中 其决速步为 $\mathrm{H}$ 原子转移及 $\mathrm{C}-\mathrm{O}$ 合环过程, 最优势的过渡 态为 TS4-1, 其对应的自由能垒为 $28.3 \mathrm{kcal} / \mathrm{mol}$. 另一方
面, 我们在计算中也考虑了其它构象的可能性, 但是它 们能量相对较高, 具体结果详见 Supporting information.

\section{$3.2 \mathrm{H}$ 转移-合环-羰基转移- $\mathrm{H}$ 转移反应机理}

接下来研究了图式 1 中显示的 path II 过程, 该反应 过程的相对自由能势能面如图 3 所示. 该机理中第一步 是由 $\mathbf{R}$ 到 $\mathbf{C} 2$ 的过程，即醇羟基的氢原子转移至 $\mathrm{N}$ 原子 及 $\mathrm{C}-\mathrm{O}$ 合环的过程. 在此应该注意到, 反应物中 $\mathrm{N}$ 原子 为 $\mathrm{sp}^{2}$ 杂化, 若要先与醇羟基的氢原子发生作用, 会使 得醇羟基的 $\mathrm{O}$ 原子带有部分负电荷, 有利于接下来的 $\mathrm{C}, \mathrm{O}$ 成键. 相对而言, 如果先发生 C-O 合环过程会使得 $\mathrm{O}$ 原子带有部分正电性, 从而导致体系的能量升高(详 见后面的讨论). 因此，我们首先考察了醇羟基的氢原 子转移至 $\mathrm{N}$ 原子的过程. 计算结果表明, 初始反应物 $\mathbf{R}$ 首先异构化为中间体 $\mathbf{R - 3}$, 其相对自由能为 $2.2 \mathrm{kcal} / \mathrm{mol}$. 随后，随着 R-3 中(醇羊基) $\mathrm{O}-\mathrm{H}$ 键的拉伸及 $\mathrm{N}, \mathrm{H}$ 原子 之间距离的缩短, 在反应路径上会得到一个氢原子转移 的过渡态 TS5. 如图 4 所示, TS5 中与羟基及亚胺相连的 两个 $\mathrm{sp}^{3}$ 杂化的 $\mathrm{C}$ 原子之间的距离也被拉伸到了 $2.043 \AA$. 说明随着 $\mathrm{O}-\mathrm{H}$ 键断裂及 $\mathrm{N}-\mathrm{H}$ 键生成, 同时伴随有 $\mathrm{C}(3)$ $-\mathrm{C}(4)$ 键的断裂而得到一个乙醛分子及中间体 M3. 随 后 M3 再与一个乙醛分子作用经过一个 $\mathrm{C}(3)-\mathrm{C}(4)$ 和 $\mathrm{C}$ -O 成键的五元环过渡态 TS6 而生成另外一个五元环 的中间体 M4, 即为图式 1 所示中间物质 C2. 由图 4 可 以看出, 在 $\mathrm{M} 4$ 中已经成键的 $\mathrm{C}-\mathrm{O}, \mathrm{C}(3)-\mathrm{C}(4)$ 之间的 距离已经由过渡态 TS6 中的 2.667, 2.202 A 缩短至 1.439 与 $1.559 \AA$. 由于 $\mathrm{M} 4$ 中存在一个特殊稳定的五元环结构 使其自由能降至 $0.1 \mathrm{kcal} / \mathrm{mol}$. 随后，M4 中的炭基基团 逐渐与五元环中的 $\mathrm{N}$ 原子靠近，同时伴随有五元环中的 $(\mathrm{Ph}-\mathrm{C}) \mathrm{C}-\mathrm{N}$ 键的拉伸而经历一个过渡态 TS7 发生羰 基基团从 $\mathrm{Ph}-\mathrm{O}$ 转移到 $\mathrm{N}$ 原子的过程. 如图 4 所示, 在 TS7 中羰基 $\mathrm{C}(2)$ 与 $\mathrm{N}$ 原子之间的距离缩短至 $1.495 \AA$, 而羰基 $\mathrm{C}(2)-\mathrm{O}(\mathrm{Ph}-\mathrm{O})$ 键被拉伸至 $2.339 \AA$. 此时五元 环中的 $(\mathrm{Ph}-\mathrm{C}) \mathrm{C}-\mathrm{N}$ 键也被拉伸至 $1.803 \AA$. 随后 $\mathrm{C}(2)$ $-\mathrm{O}$ 键完全断裂，羰基 $\mathrm{C}(2)-\mathrm{N}$ 键生成而得到一个相对 自由能为 $6.0 \mathrm{kcal} / \mathrm{mol}$ 的中间体 M5. 在 M5 中原来五元 环中的 $(\mathrm{Ph}-\mathrm{C}) \mathrm{C}-\mathrm{N}$ 键被拉伸至 $2.982 \AA$ ，此数据表明 在羰基转移过程中会伴随有 $\mathrm{C}-\mathrm{N}$ 键的开环行为. 最后 中间体 $M 5$ 再经过一个 $\mathrm{H}$ 原子转移及 $\mathrm{C}-\mathrm{N}$ 键重新合环 的协同反应过渡态 TS8, 最终得到产物 P. 根据图 4, 整 个 $\mathrm{H}$ 转移-合环-羰基转移 $-\mathrm{H}$ 转移机理中决速步骤是 $\mathrm{C}$ $-\mathrm{C}$ 与 $\mathrm{C}-\mathrm{O}$ 键合环步骤, 其对应的总活化能垒为 40.1 $\mathrm{kcal} / \mathrm{mol}$.

为了尽可能地将所有的反应机理考虑在内, 本课题 组又计算了 $\mathbf{B} \rightarrow \mathbf{C 2}$ 过程中先发生 C-O 合环的情况. 该 过程我们没有得到相应过渡态. 通过固定要成键的 $\mathrm{C}-$ $\mathrm{O}$ 之间的不同距离得到了该反应路径的势能面能量扫 描图(见 supporting information 图 S4), 我们发现 $\mathrm{C}-\mathrm{O}$ 


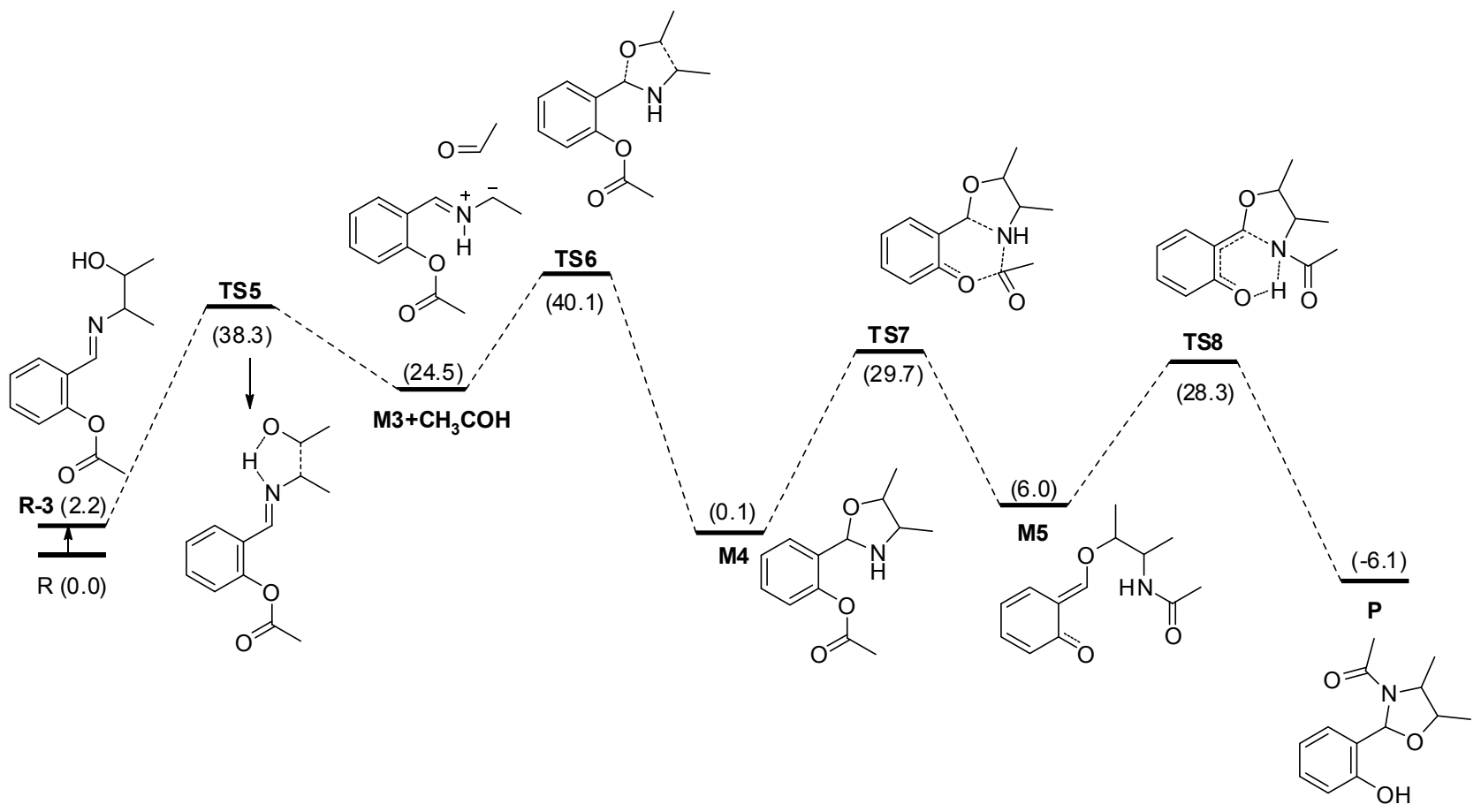

图 $3 \mathrm{H}$ 转移-合环-羰基转移- $\mathrm{H}$ 转移反应中的势能面图(图中的数据为吉布斯自由能, 单位 $\mathrm{kcal} / \mathrm{mol}$ )

Figure 3 Energy profile of $\mathrm{H}$ transfer-cyclization-carbonyl transfer-H transfer reaction (the values are Gibbs free energies in $\mathrm{kcal} / \mathrm{mol}$ )
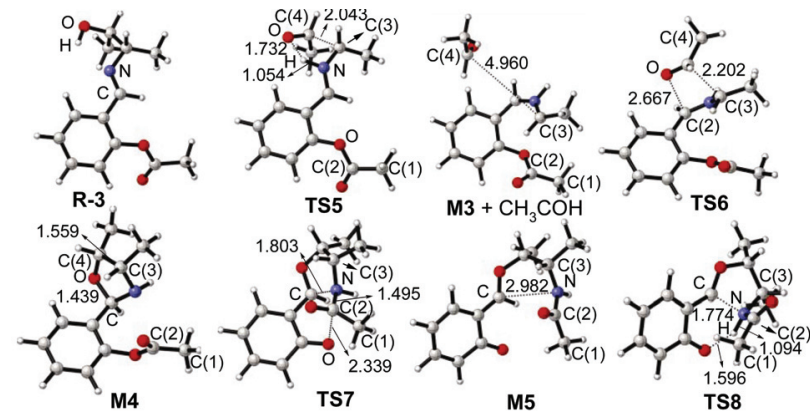

图 $4 \mathrm{H}$ 转移-合环-羰基转移- $\mathrm{H}$ 转移反应中各物质的优化构型(键长 单位为 $\AA$ )

Figure 4 Optimized structures of species in $\mathrm{H}$ transfer-cyclizationcarbonyl transfer- $\mathrm{H}$ transfer reaction (bond lengths are given in $\AA$ )

之间的距离由 $3.2 \AA$ 逐步变化到 $1.5 \AA$ 过程中体系的能 量在不断升高. 体系能量相对变化值为 $65 \mathrm{kcal} / \mathrm{mol}$ 左右. 这么高的能量表明, 室温下此反应路径的发生在整个反 应体系中所占的几率很低.

随后，又计算了接下来由中间物 M4 开始先经历 N 原子上的 $\mathrm{H}$ 原子转移到 $\mathrm{Ph}-\mathrm{O}$ 原子的过程, 结果也没有 找到对应的过渡态. 按照相同的方法, 通过固定不同的 $(\mathrm{Ph}-\mathrm{O}) \mathrm{O}-\mathrm{H}$ 之间的距离得到了体系的势能面能量变 化情况(见 supporting information 图 S5). 扫描的 $\mathrm{O}-\mathrm{H}$ 之间的长度范围为 $1.11 \sim 1.97 \AA$. 在此变化过程中体系 的能量升高了 $49 \mathrm{kcal} / \mathrm{mol}$ 左右. 因此该过程在室温下对 整个反应体系的影响也是非常小的，可以忽略.

\section{3 两种反应机理的对比}

由前面的讨论得知, $B \rightarrow C 1 \rightarrow D$ 的反应路径存在一
个羰基转移-合环的机理, 而 $\mathbf{B} \rightarrow \mathbf{C} \mathbf{2} \rightarrow \mathbf{D}$ 过程按照 $\mathrm{H}$ 转 移-合环-羰基转移- $\mathrm{H}$ 转移的机理进行. 两条不同路径 的反应物和产物相同, 因此不同机理的相对优劣主要由 动力学决定. $\mathbf{B} \rightarrow \mathbf{C 1} \rightarrow \mathbf{D}$ 的转化过程决速步为 $\mathrm{H}$ 原子转 移与 C-O 成环步骤, 其总活化能垒是 $28.3 \mathrm{kcal} / \mathrm{mol}$; 而 $\mathrm{H}$ 转移-合环-羰基转移- $\mathrm{H}$ 转移机理中反应的决速步是 $\mathrm{C}-\mathrm{C}$ 及 $\mathrm{C}-\mathrm{O}$ 成键的合环步骤, 其总活化能垒高达 40.1 $\mathrm{kcal} / \mathrm{mol}$. 应该注意到这一结论与 Wong 等此前的研究 有所不同，其原因在于本文中在模型反应中保留了亚胺 基，而非对其进行- $\mathrm{NH}_{2}$ 模型化; 另一方面，本文对由中 间体 B 到产物 D 的过程进行了全面系统讨论, 而此前 Wong 等研究中主要针对四肽环化步骤与传统的直接酰 胺键成键步骤的相对难易.

我们分析了羰基转移-合环机理相对 $\mathrm{H}$ 转移-合环羰基转移 $-\mathrm{H}$ 转移机理优势的原因. 我们认为在羰基转 移一合环机理中, 决速步 $\mathrm{H}$ 原子转移与 $\mathrm{C}-\mathrm{O}$ 成环的过程 中只有一个 $\mathrm{O}-\mathrm{H}$ 键的断裂且同时会伴随有 $\mathrm{C}-\mathrm{O}, \mathrm{O}-$ $\mathrm{H}$ 两个价键的生成. 然而在 $\mathrm{H}$ 转移一合环-羰基转移- $\mathrm{H}$ 转 移反应机理中，体系无论是发生 $\mathrm{H}$ 原子的转移还是发生 羰基的转移，在一个价键生成的同时会伴随有两个价键 的断裂. 因此体系需要更多的能量才能发生反应. 故在 肽环化的两个反应历程中需要能量较小的羰基转移一合 环反应机理占据主导地位.

此外, 为了验证以上结论的可行性, 我们还运用另 外两种不同的密度泛函理论方法 TPSS 及 MPWB1K 计 算了不同体系内各中间体及过渡态能量. 表 1 给出了在 三种不同方法下计算得到的反应体系中各物质的相对 
吉布斯自由能值. 从表 1 中的数据可以看出, 在 MPWB1K 水平上得到的各物质的相对吉布斯自由能变 化值与 M06 方法得到的结果相接近. 而用 TPSS 方法计 算得到的结果稍低. 但三种方法得到的结论一致, 即协 同机理(Path I)相对分步机理(Path II)在能量上更为优势, 而且协同机理的决速步为 $\mathrm{H}$ 原子转移及 $\mathrm{C}-\mathrm{O}$ 成环步骤.

表 1 在不同理论水平上计算得到的反应体系中各物质的相对吉布斯 自由能 $\left[\Delta G /\left(\mathrm{kcal} \cdot \mathrm{mol}^{-1}\right)\right]$

Table 1 The relative Gibbs values $\left[\Delta G /\left(\mathrm{kcal}^{\circ} \mathrm{mol}^{-1}\right)\right]$ of species in the studied system at different levels

\begin{tabular}{|c|c|c|c|c|}
\hline Species & & $\Delta G^{a}$ & $\Delta G^{b}$ & $\Delta G^{c}$ \\
\hline \multirow{14}{*}{$\begin{array}{l}\text { Carbonyl } \\
\text { mechanism }\end{array}$} & $\mathbf{R}$ & 0.0 & 0.0 & 0.0 \\
\hline & R-1 & 1.8 & 1.7 & 1.2 \\
\hline & R-2 & 6.0 & 3.4 & 4.3 \\
\hline & TS1 & 20.2 & 15.6 & 22.0 \\
\hline & TS2 & 16.4 & 11.3 & 16.6 \\
\hline & TS3 & 62.7 & 54.4 & 67.3 \\
\hline & M2-1 & 17.0 & 14.7 & 19.7 \\
\hline & M2-2 & 13.2 & 10.4 & 14.3 \\
\hline & M2-3 & 24.5 & 22.7 & 24.9 \\
\hline & M2-4 & 20.4 & 20.2 & 22.1 \\
\hline & TS4 & 32.0 & 25.3 & 32.4 \\
\hline & TS4-1 & 28.3 & 21.7 & 27.8 \\
\hline & P-1 & 3.1 & 4.6 & -0.3 \\
\hline & Prod & -6.1 & -5.2 & -10.6 \\
\hline \multirow{8}{*}{$\begin{array}{l}\mathrm{H} \text { transfer-cyclization-carbonyl } \\
\text { transfer-H transfer mechanism }\end{array}$} & $\mathbf{R}-3$ & 2.2 & -0.9 & 0.0 \\
\hline & TS5 & 38.3 & 26.9 & 41.7 \\
\hline & $\mathbf{M 3}+\mathrm{CH}_{3} \mathrm{COH}$ & 24.5 & 22.7 & 24.9 \\
\hline & TS6 & 40.1 & 30.9 & 44.7 \\
\hline & M4 & 0.1 & 0.9 & -3.9 \\
\hline & TS7 & 29.7 & 24.7 & 28.1 \\
\hline & M5 & 6.0 & 2.6 & 7.0 \\
\hline & TS8 & 28.3 & 23.3 & 26.2 \\
\hline
\end{tabular}

${ }^{a}$ at the M06/6-311++G(2df,2p) level; ${ }^{b}$ at the TPSS/6-311++G(2df,2p) level; ${ }^{c}$ at the MPWB1K/6-311++G(2df,2p) level.

\section{4 结论}

采用 M06/6-311++G(2df,2p)//B3LYP/6-31G(d)方 法对四肽环化过程中存在的两个不同的反应历程进行 详细的理论研究. 计算结果表明, 由亚胺到环肽过程中 存在两种不同的反应机理. 一个是先经历羊基转移, 然 后再发生 $\mathrm{H}$ 转移与 $\mathrm{C}-\mathrm{O}$ 合环协同进行的羰基转移一合环 反应机理. 另一个是先 $\mathrm{H}$ 原子转移生成一个中间体, 然 后再进行 $\mathrm{C}-\mathrm{O}$ 合环, 随后再进行羰基转移与 $\mathrm{H}$ 原子转移 的分步进行的 $\mathrm{H}$ 转移一合环一羰基转移- $\mathrm{H}$ 转移反应机制. 羰基转移一合环机理与 $\mathrm{H}$ 转移一合环-羰基转移 $-\mathrm{H}$ 转移机 理的决速分别是 $\mathrm{H}$ 原子转移与 $\mathrm{C}-\mathrm{O}$ 成环协同步骤以及 $\mathrm{C}-\mathrm{C}$ 与 $\mathrm{C}-\mathrm{O}$ 键合环协同步骤, 其总活化能垒分别是
28.3 及 $40.1 \mathrm{kcal} / \mathrm{mol}$. 羰基转移一合环反应历程中所需要 克服的自由能垒要小于 $\mathrm{H}$ 转移-合环-羰基转移- $\mathrm{H}$ 转移 反应过程. 因此在肽环化过程中羰基转移一合环反应机 理占主导地位.

\section{致谢}

本文中所有计算均在中国科学技术大学超级计算 中心及上海超级计算中心完成并受中国教育科研网格 ChinaGrid 资助.

\section{References}

[1] (a) Guani-Guerra, E.; Santos-Mendoza, T.; Lugo-Reyes, S. O.; Terán, L. M. Clin. Immunol. 2010, 135, 1. (b) Badawi, A. H.; Siahaan, T. J. Clin. Immunol. 2012, 144, 127. (c) Hartgerink, J. D.; Granja, J. R.; Milligan, R. A.; Ghadiri, M. R. J. Am. Chem. Soc. 1996, $118,43$.

[2] Ovchinnikov, Y. A.; Ivanov, V. T. Tetrahedron 1975, 31, 2177.

[3] (a) Driggers, E. M.; Hale, S. P.; Lee, J.; Terrett, N. K. Nat. Rev. Drug. Discovery 2008, 7, 608. (b) Craik, D. J.; Allewell, N. M. J. Biol. Chem. 2012, 287, 26999.

[4] (a) Jiang, S.; Li, Z.; Ding, K.; Roller, P. Curr. Org. Chem. 2008, 12 , 1502. (b) Tam, J. P.; Wong, C. T. T. J. Biol. Chem. 2012, 287, 27020.

[5] White, C. J.; Yudin, A. K. Nat. Chem. 2011, 3, 509.

[6] (a) Wasserman H. H.; Zhang, R. Tetrahedron Lett. 2002, 43, 3743. (b) Nilsson, B. L.; Soellner, M. B.; Raines, R. T. Annu. Rev. Biophys. Biomol. Struct. 2005, 34, 91. (c) Li, X.; Lam, H. Y.; Zhang, Y.; Chan, C. K. Org. Lett. 2010, 12, 1724. (d) Clark, R. J.; Craik, D J. Biopolymers 2010, 94, 414. (e) Tam, J. P.; Wong, C. T. T. J. Biol. Chem. 2012, 287, 27020. (f) Zhao, J.-F.; Zhang, X.-H.; Ding, Y.-J.; Yang, Y.-S.; Bi, X.-B.; Liu, C.-F. Org. Lett. 2013, 15, 5182. (g) Cui, H.-K.; Guo, Y.; He, Y.; Wang, F.-L.; Chang, H.-N.; Wang, Y.-J.; Wu, F.-M.; Tian, C.-L.; Liu, L. Angew. Chem., Int. Ed. 2013, 52, 9558. (h) Tang, S.; Zheng, J.-S.; Yang, K.; Liu, L. Acta Chim. Sinica 2012, 70, 1471.(唐珊, 郑基深, 杨可, 刘否, 化学学报, 2012, 70, 1471.)

[7] Wong, C. T. T.; Lam, H. Y.; Song, T.; Chen, G.; Li, X. Angew. Chem., Int. Ed. 2013, 52, 10212.

[8] (a) Zhang, Y.; Xu, C.; Lam, H. Y.; Lee, C. L.; Li, X. Proc. Natl. Acad. Sci. U. S. A. 2013, 110, 6657. (b) Lam, H. Y.; Zhang, Y.; Liu, H.; Xu, J.; Wong, C. T.; Xu, C.; Li, X. J. Am. Chem. Soc. 2013, 135, 6272. (c) Li, X.; Lam, H. Y.; Zhang, Y.; Chan, C. K. Org. Lett. 2010, 12, 1724

[9] (a) Torbeev, V. Y.; Raghuraman, H.; Mandal, K.; Senapati, S.; Perozo, E.; Kent, S. B. H. J. Am. Chem. Soc. 2009, 131, 884. (b) Aucagne, V.; Valverde, I. E.; Marceau, P.; Galibert, M.; Dendane, N.; Delmas, A. F. Angew. Chem., Int. Ed. 2012, 51, 11320. (c) Huang, Y. C.; Li, Y. M.; Chen, Y.; Pan, M.; Li, Y. T.; Yu, L.; Guo, Q. X.; Liu, L. Angew. Chem., Int. Ed. 2013, 52, 4858. (d) Zhan, C. Y.; Varney, K.; Yuan, W. R.; Zhao, L.; Lu, W. Y. J. Am. Chem. Soc. 2012, 134, 6855. (e) Fang, G. M.; Wang, J. X.; Liu, L. Angew. Chem., Int. Ed. 2012, 51, 10347. (f) McGinty, R. K.; Kim, J.; Chatterjee, C.; Roeder, R. G.; Muir, T. W. Nature 2008, 453, 812. (g) Tan, Z.; Shang, S.; Danishefsky, S. J. Angew. Chem., Int. Ed. 2010, 49, 9500. (h) Fang, G. M.; Li, Y. M.; Shen, F.; Huang, Y. C.; Li, J. B.; Lin, Y.; Cui, H. K.; Liu, L. Angew. Chem., Int. Ed. 2011, 50 , 7645. (i) Kumar, K. S.; Bavikar, S. N.; Spasser, L.; Moyal, T.; Ohayon, S.; Brik, A. Angew. Chem., Int. Ed. 2011, 50, 6137. (j) Li, Y.-M.; Li, Y.-T.; Pan, M.; Kong, X.-Q.; Huang, Y.-C.; Hong, Z.-Y.; Liu, L. Angew. Chem., Int. Ed. 2014, 53, 2198. (k) Zheng, J.-S.; Tang, S.; Qi, Y.-K.; Wang, Z.-P.; Liu, L. Nat. Protoc. 2013, 8, 2483.

[10] (a) Lee, C.; Yang, W.; Parr, R. G. Phys. Rev. B 1988, 37, 785. (b) Becke, A. D. J. Chem. Phys. 1993, 98, 5648.

[11] (a) Peyghan, A. A.; Noei, M. Comput. Mater. Sci. 2014, 82, 97. (b) Rajak, K.; Maiti, B. J. Chem. Phys. 2014, 140, 44314. (c) Li, W.-Z.; Yan, B.-F.; Xiao, C.-P.; Li, Q.-Z.; Cheng, J.-B. J. Organomet. Chem. 2014, 750, 112. (d) Buceta, N. N.; Ruiz, D.; Romaneli, G. P.; Autino, J. C.; Duddeck, H.; Diez, R. P.; Jios, J. L. J. Phys. Org. 
Chem. 2014, 27, 106. (e) Milovanovic, M. Z.; Jerosimic, S. V. Int. J. Quantum Chem. 2014, 114, 192. (f) Auzmendi-Murua, I.; Bozzelli, J. W. Int. J. Chem. Kinet. 2014, 46, 71. (g) Annaraj, B.; Pan, S.; Neelakantan, M. A.; Chattaraj, P. K. Comput. Theor. Chem. 2014, 1028, 19. (h) Uzunova, E. L.; Mikosch, H. J. Chem. Phys. 2014, 140, 024303. (i) Tang, S.-Y.; Fu, Y.; Guo, Q.-X. Acta Chim. Sinica 2012，70，1923. (唐诗雅，傅尧，郭庆祥，化学学报，2012，70, 1923.) (j) Li, J.; Xu, W.-L.; Hu, J.; Ling, M.; Yao, J.-H. Acta Phys.-Chim. Sin. 2013, 29, 1923. (李佳, 徐雯利, 胡静, 凌敏, 姚 建华，物理化学学报, 2013, 29, 1923.) (k) Zheng, W.-R.; Fu, Y.; Wang, H. J.; Guo, Q. X. Chin. J. Org. Chem. 2008, 28, 459. (郑文 锐，傅尧，王华静，郭庆祥，有机化学， 2008，28，459.) (1) Ai-Fifi, Z.; Eid, M.; Saleh, N. A.; Lbrahim, M. J. Comput. Theor. Nanos. 2014, 11, 409. (m) Zhu, X. Q.; Chen, X.; Mei, L.-R. Org. Lett. 2011, 13, 2456. (n) Yu, H.; Yang, Y.; Zhang, L.; Dang, Z.; Hu, G. J. Phys. Chem. A 2014, 118, 606. (o) Zhang, Q.; Yu, H.-Z.; Shi, J. Acta Phys.-Chim. Sin. 2013, 29, 2321. (张琪, 于海珠, 石景, 物理化学 学报, 2013, 29, 2321.)

[12] (a) Zhao, Y.; Truhlar, D. G. Phys. Chem. Chem. Phys. 2005, 7, 2701. (b) Senthilnithy, R.; Weerasingha, M. S. S.; Dissanayake, D. P. Comput. Theor. Chem. 2014, 1028, 60.

[13] (a) Fukui, K. Acc. Chem. Res. 1981, 14, 363. (b) Fukui, K. J. Phys. Chem. 1970, 74, 4161.

[14] (a) Zhao, Y.; Truhlar, D. G. Theor. Chem. Acc. 2008, 120, 215. (b) Walker, M.; Harvey, A. J. A.; Sen, A.; Dessent, C. E. H. J. Phys. Chem. A 2013, 117, 12590. (c) Yu, H.; Fu, Y. Chem. Eur. J. 2012, 18, 16765. (d) Zhang, Q.; Yu, H.-Z.; Fu, Y. Organometallics 2013, 32, 4165. (e) Liu, D.-J.; Yu, H.-Z.; Fu, Y. Acta Chim. Sinica 2013, 71,1385 . (刘丁嘉, 于海珠, 傅尧, 化学学报, 2013, 71, 1385.)

[15] (a) Perdew, J. P.; Wang, Y. Phys. Rev. B 1992, 45, 13244. (b) Tao,
J.; Perdew, J. P.; Staroverov, V. N.; Scuseria, G. E. Phys. Rev. Lett. 2003, 91, 146401.

[16] Zhao, Y.; Truhlar, D. G. J. Phys. Chem. A 2004, 108, 6908.

[17] Marenich, A. V.; Cramer, C. J.; Truhlar, D. G. J. Phys. Chem. B 2009, 113, 6378 .

[18] Frisch, M. J.; Trucks, G. W.; Schlegel, H. B.; Scuseria, G. E.; Robb, M. A.; Cheeseman, J. R.; Scalmani, G.; Barone, V.; Mennucci, B.; Petersson, G. A.; Nakatsuji, H.; Caricato, M.; Li, X.; Hratchian, H. P.; Izmaylov, A. F.; Bloino, J.; Zheng, G.; Sonnenberg, J. L.; Hada, M.; Ehara, M.; Toyota, K.; Fukuda, R.; Hasegawa, J.; Ishida, M.; Nakajima, T.; Honda, Y.; Kitao, O.; Nakai, H.; Vreven, T.; Montgomery, J. A., Jr.; Peralta, J. E.; Ogliaro, F.; Bearpark, M.; Heyd, J. J.; Brothers, E.; Kudin, K. N.; Staroverov, V. N.; Kobayashi, R.; Normand, J.; ghavachari, K.; Rendell, A.; Burant, J. C.; Iyengar, S. S.; Tomasi, J.; Cossi, M.; Rega, N.; Millam, J. M.; Klene, M.; Knox, J. E.; Cross, J. B.; Bakken, V.; Adamo, C.; Jaramillo, J.; Gomperts, R.; Stratmann, R. E.; Yazyev, O.; Austin, A. J.; Cammi, R.; Pomelli, C.; Ochterski, J. W.; Martin, R. L.; Morokuma, K.; Zakrzewski, V. G.; Voth, G. A.; Salvador, P.; Dannenberg, J. J.; Dapprich, S.; Daniels, A. D.; Farkas, Foresman, J. B.; Ortiz, J. V.; Cioslowski, J.; Fox, D. J. Gaussian 09, Revision B. 01; Gaussian, Inc., Wallingford, CT, 2009.

[19] (a) Zheng, J. S.; Tang, S.; Huang, Y. C.; Liu, L. Acc. Chem. Res. 2013, 46, 2475. (b) Sharma, R.; Tam, J. P. Org. Lett. 2011, 13, 5176. (c) Hou, W.; Zhang, X.; Li, F.; Liu, C. F. Org. Lett. 2011, 13, 386. (d) Zheng, J. S.; Chang, H. N.; Wang, F. L.; Liu, L. J. Am. Chem. Soc. 2011, 133, 11080. (e) Ollivier, N.; Dheur, J.; Mhidia, R.; Blanpain, A.; Melnyk, O. Org. Lett. 2010, 12, 5238. (f) Botti, P.; Villain, M.; Manganiello, S.; Gaertner, H. Org. Lett. 2004, 6, 4861. 